\title{
Diacronie
}

Studi di Storia Contemporanea

$\mathrm{N}^{\circ} 25,1 \mid 2016$

"Se creare è definire"

\section{Claudio Mancuso, La patria in festa. Ritualità pubblica e religioni civili in Sicilia (1860-1911)}

\section{Deborah Paci}

\section{(2) OpenEdition \\ 1 Journals}

\section{Edizione digitale}

URL: http://journals.openedition.org/diacronie/3893

DOI: 10.4000/diacronie.3893

ISSN: 2038-0925

Editore

Association culturelle Diacronie

Notizia bibliografica digitale

Deborah Paci, « Claudio Mancuso, La patria in festa. Ritualità pubblica e religioni civili in Sicilia (1860-1917) », Diacronie [Online], № 25, 1 | 2016, documento 13, Messo online il 29 mars 2016,

consultato il 23 septembre 2020. URL : http://journals.openedition.org/diacronie/3893 ; DOI : https:// doi.org/10.4000/diacronie.3893 


\title{
Diacronie
}

N. 25 | $1 \mid 2016$ "Se creare è definire"

13/

\section{RECENSIONE:}

\section{Claudio MANCUSO, La patria in festa.} Ritualità pubblica e religioni civili in Sicilia (186o-1911), Palermo, Edizioni La Zisa, 2013, 460 pp.

a cura di Deborah PACI *

Claudio Mancuso è docente a contratto di Storia Contemporanea presso l'Università degli Studi di Palermo e l'Università degli Studi di Torino; La patria in festa è frutto del lavoro di ricerca portato avanti durante il corso di laurea specialistica e il dottorato, conseguito presso l'Università di Urbino.

Il libro indaga come è stato "raccontato" il Risorgimento in Sicilia: questo filone di studi ha sinora, colpevolmente, trascurato il Meridione.

\begin{abstract}
Attraverso il filtro della festa è possibile osservare in che modo le parti politiche procedono all'occupazione simbolica del tempo e dello spazio, come la ritualità pubblica riesce a influenzare la percezione sociale e politica degli individui e in che misura i processi di rappresentazione del potere pervengono alla definizione di una sensibilità comunitaria e identitaria condivisa ${ }^{1}$.
\end{abstract}

Mancuso intende indagare la periferia del paese e, insieme ad essa la miriade di borghi semirurali sovrarappresentati dall'indagine storica. Il periodo preso in esame è il primo cinquantennio unitario, quello "liberale". L'autore ha privilegiato, nell'uso delle fonti, il ricorso a deliberazioni e provvedimenti delle autorità e delle amministrazioni locali dell'isola; è stato inoltre fatto ricorso alla documentazione proveniente dalle Società di Storia Patria di Palermo, Catania e Messina e alla stampa nazionale e locale.

\footnotetext{
${ }^{1}$ MANCUSO, Claudio, La patria in festa. Ritualità pubblica e religioni civili in Sicilia (18601911), Palermo, Edizioni La Zisa, 201, pp. 27-28.
} 
La ricerca di Mancuso ruota intorno a tre assi principali, che offrono una suddivisione del libro:

La ricerca è stata articolata intorno a tre nuclei fondamentali [...]. Nella prima parte è stato definito il contesto politico, sociale ed economico della Sicilia postunitaria, all'interno del quale le strategie di pedagogia patriottica trovarono attuazione e sviluppo concreti [...]. Nella seconda parte è stato preso in considerazione il tema delle modalità per mezzo delle quali le celebrazioni nazionali si inserirono nel quadro festivo preesistente, caratterizzato da tradizioni secolari civili e religiose, ovvero in quale misura lo modificarono, e in che termini, invece, ne fagocitarono forme, simboli e contenuti. [...] Nella terza parte della ricerca, l'attenzione è stata focalizzata sull'analisi degli strumenti mediante i quali la nuova festa diventò essa stessa tradizione, seguendo la concreta articolazione del nuovo repertorio rituale immesso dalle celebrazioni associate alla nuova religione laica ${ }^{2}$.

Mancuso fornisce un quadro introduttivo delle condizioni della Sicilia e dell'atteggiamento tenuto dal governo centrale nei confronti dell'Isola e del mutamento di classe dirigente verificatosi durante l'età liberale. Per avere un'idea del mutamento avvenuto con il passaggio allo stato sabaudo, basta prendere in considerazione come con il passaggio al nuovo regime si verificò un'equiparazione fra le città: Palermo perse le prerogative di capitale, ritrovandosi ridotta al rango delle altre città siciliane.

Nel secondo capitolo l'autore si sofferma sulle metamorfosi della ritualità della festa. La premessa di Mancuso è che «l'utilizzo del dispositivo della festa in chiave di legittimazione politica e di consolidamento dell'ordine sociale esistente non costituì una peculiarità esclusiva del nuovo governo liberale»3. Mancuso sottolinea una duplice tendenza, solo apparentemente contraddittoria, sorta negli anni immediatamente successivi all'Unità: da una parte l'abolizione delle tradizioni antiche, soprattutto religiose, in modo da suggellare la rottura con il passato; dall'altra la necessità di non offendere i sentimenti popolari. Riguardo alla modalità delle celebrazioni nazionali, Mancuso rileva una persistenza più duratura dell'antico sistema di valori; tuttavia la manifestazione vide qualche mutamento:

Nondimeno, la festa popolare e religiosa, seppur svuotata del suo contenuto originario e ridotta nelle manifestazioni esteriori, continua ad avere un peso e un ruolo cruciali nella mentalità e nella cultura dei siciliani, che in molte circostanze si

\footnotetext{
${ }^{2}$ Ibidem, pp. 31-33.

3 Ibidem, p. 65. 
opposero, e anche efficacemente, alla politica di soppressione delle festività tradizionali promossa dal nuovo Stato. Tuttavia, esse furono costrette a cambiare pelle, a scendere a patti con le esigenze pedagogiche e culturali della giovane compagine nazionale, e persero, così, una parte, più o meno consistente, delle connotazioni folkloriche e degli aspetti tipici della religiosità d'antico regime per assumere invece i tratti delle moderne identità borghesi e popolari4.

L'unica soluzione percorribile era quindi quella che prevedeva una graduale modificazione delle festività, funzionale a introdurre progressivamente la simbologia patriottica. Il repertorio simbolico venne quindi utilizzato nel quadro di nuovi significati:

il ricco emporio di uomini illustri, che, fino alle soglie dell'Unità, costituirono le principali espressioni dell'identità municipale, fu reinterpretato in chiave nazionale. I grandi morti del passato divennero i patroni laici della nuova collettività urbana, e una tradizione eminentemente cittadina fu compresa entro un quadro più ampio e ispirato ai principi dell'unità e dell'indipendenza nazionali5.

Nel terzo capitolo Mancuso si sofferma proprio sull'utilizzo di figure di letterati e artisti in chiave "nazionalizzatrice" in Sicilia, concentrandosi in particolar modo su Dante.

Nella parte terza del libro, l'autore mette quindi in evidenza la necessità per la monarchia di nazionalizzarsi per essere in grado di legare alle istituzioni tutti gli italiani. Risulta di particolare interesse il caso dello Statuto, preso in esame perché le celebrazioni di questa solennità vennero affidate all'iniziativa delle singole realtà periferiche.

In una fase di transizione e adattamento alle nuove istituzioni, la festa nazionale assunse una funzione unificatrice, di collante identitario, suscitando una forte spinta partecipativa e ottenendo un ampio coinvolgimento sia delle istituzioni locali, sia della cittadinanza ${ }^{6}$.

A partire dagli anni Ottanta dell'Ottocento si andò incontro a un congelamento del rituale che, se da una parte ne ridusse la partecipazione, dall'altro rappresentò un passo avanti nella creazione di una tradizione.

4 Ibidem, p. 405.

5 Ibidem, p. 101.

${ }^{6}$ Ibidem, p. 182. 
Se queste prime feste permettevano di trovare un'intesa con il clero, la celebrazione del 20 settembre - insediamento del re a Roma - portò, inevitabilmente, a uno scontro con la Chiesa. Ma uno dei passi più importanti per l'attecchimento di una pedagogia patriottica fu fatto con l'adozione della figura di Garibaldi, specificamente durante la fase di governo della Sinistra storica, dal momento che Garibaldi era «riuscito a ottenere un livello di consenso decisamente insolito per l'Italia dell'Ottocento»7 e questo per via del fatto che «era la personificazione del popolo, l'uomo che, con la sua iniziativa e il suo coraggio aveva reso possibile l'unificazione del paese e che, a buon diritto, campeggiava con Vittorio Emanuele II nel pantheon degli eroi e dei padri della patria» 8 .

Ed è proprio al culto di Garibaldi e dei Mille che è dedicato il capitolo cinque; nondimeno ma alla celebrazione dell'epopea garibaldina si aggiunsero tutta una serie di eventi celebrativi di personaggi considerati come sussidiari o complementari al Risorgimento. Fu questo il caso dell'inaugurazione della statua di Crispi nel capoluogo siciliano, descritto da Mancuso in questi termini: «probabilmente mai nessuna cerimonia patriottica a Palermo aveva coinvolto così tanta gente»9. L'evento - ritenuto l'ideale suggello dell'età risorgimentale - vide il trasferimento della salma di Crispi, imbalsamata e in virtù di ciò «in perfetto stato di conservazione» ${ }^{10}$. La cerimonia, benché suddivisa in una parte religiosa ed una civile, sembrava ricollegarsi all'idea di traslazione di un santo più che di uno statista, come dimostra l'attenzione al particolare dell'incorruzione del corpo.

Il sesto capitolo prende invece in esame le cerimonie messe in opera dalle opposizioni o comunque non riconducibili alle ideologie ufficiali. Queste, infatti, «portarono avanti un processo di costruzione e promozione di una visione alternativa delle lotte risorgimentali» ${ }^{11}$.

Come sottolinea Mancuso:

la pericolosità di queste celebrazioni , rispetto al mondo liberale, risiedeva non tanto nella promozione di un immaginario simbolico minoritario, quanto nella prefigurazione di un sistema, non soltanto ideologico, ma anche politico e istituzionale, alternativo a quello monarchico-liberale ${ }^{12}$.

\footnotetext{
7 Ibidem, p. 225.

8 Ibidem.

9 Ibidem, p. 308.

${ }^{10}$ Ibidem, p. 307.

${ }^{11}$ Ibidem, p. 313.

${ }_{12}$ Ibidem, p. 314.
} 
Il lavoro è risultato, per l'autore, molto più difficile a causa del limitato numero di fonti disponibili; in questo caso le notizie sono state reperite nelle testate legate agli ambienti estremisti o nei verbali delle questure e prefetture.

Mazzini, ad esempio, divenne uno dei personaggi di riferimento per l'estremismo, in virtù del suo rappresentare «una sfida al sistema liberale in quanto proposta di un modello alternativo» ${ }^{13}$.

Un'altra delle ragioni d'opposizione era quella che faceva leva sul concetto di "Risorgimento incompleto". In quest'ottica risultò fondamentale la ripresa di alcune figure, come quella di Guglielmo Oberdan, con intenti irredentisti.

Altri temi cari al radicalismo furono la tradizione rivoluzionaria francese e la festa del Primo maggio, trattati anch'essi nel sesto capitolo.

Nelle conclusioni Mancuso tira le fila di quelli che furono i mutamenti nella concezione di festa in Sicilia nel passaggio dalla società di antico regime a quella borghese.

La chiave di lettura che ci offre l'autore è che:

la questione della costruzione dell'identità nazionale va letta inevitabilmente nel quadro di una sempre più accesa lotta per il controllo del potere politico, e la continua dinamica di opposizione e conciliazione tra i simboli del mito ufficiale e le icone del mito di opposizione rappresentò l'elemento distintivo del processo di costruzione della nuova tradizione patriottica nazionale ${ }^{14}$.

L'intento di Mancuso è quello di dimostrare come non vi fosse una grande distanza nella manifestazione pubblica del sentimento nazionale patriottico tra la Sicilia e le regioni centrosettentrionali. L'auspicio dell'autore è quello che la linea di ricerca da lui tracciata possa essere affiancata sia da altri studi sulla ritualità della festa in Sicilia, sia nelle altre regioni del Sud.

13 Ibidem, p. 320.

14 Ibidem, p. 411. 


\section{* L'autore}

Deborah Paci è assegnista di ricerca presso il Dipartimento di Studi linguistici e culturali comprati dell'Università Ca' Foscari di Venezia. È autrice della monografia Corsica fatal, Malta baluardo di romanità. L’ìredentismo fascista nel mare nostrum (1922-1942), Firenze-Milano, Le Monnier-Mondadori, 2015.

URL: < http://www.studistorici.com/2009/o2/24/deborah-paci/ >

\section{Per citare questo articolo:}

PACl, Deborah, «Recensione: Claudio MANCUSO, La patria in festa. Ritualità pubblica e religioni civili in Sicilia (1860-1911), Palermo, Edizioni La Zisa, 2013, 460 pp.», Diacronie. Studi di Storia Contemporanea : "Se creare è definire", 29/03/2016,

URL:<http://www.studistorici.com/2016/03/29/paci_numero_25/ >

\section{Diacronie Studi di Storia Contemporanea 3 www.diacronie.it}

Risorsa digitale indipendente a carattere storiografico. Uscita trimestrale. redazione.diacronie@hotmail.it

Comitato di redazione: Jacopo Bassi - Luca Bufarale - Elisa Grandi - Antonio César Moreno Cantano - Deborah Paci - Fausto Pietrancosta - Alessandro Salvador - Matteo Tomasoni - Luca Zuccolo

Diritti: gli articoli di Diacronie. Studi di Storia Contemporanea sono pubblicati sotto licenza Creative Commons 3.0. Possono essere riprodotti e modificati a patto di indicare eventuali modifiche dei contenuti, di riconoscere la paternità dell'opera e di condividerla allo stesso modo. La citazione di estratti è comunque sempre autorizzata, nei limiti previsti dalla legge. 